Submitted version of of: W. P. du Plessis, C. Kitching and A. bin Ghannam, "Element Sharing in Interleaved Antenna Arrays," 6 ${ }^{\text {th }}$ European Conference on Antennas and Propagation (EuCAP), 26-30 March 2012. The published version will be available online at: http://ieeexplore.iee.org/arnumber=6206639

(c) 2012 IEEE. Personal use of this material is permitted. Permission from IEEE must be obtained for all other uses, in any current or future media, including reprinting/republishing this material for advertising or promotional purposes, creating new collective works, for resale or redistribution to servers or lists, or reuse of any copyrighted component of this work in other works. 


\title{
Element Sharing in Interleaved Antenna Arrays
}

\author{
W. P. du Plessis ${ }^{\dagger}$, C. Kitching ${ }^{\dagger}$ and A. bin Ghannam* \\ $\dagger$ Defence, Peace, Safety and Security (DPSS) \\ Council for Scientific and Industrial Research (CSIR) \\ Pretoria, South Africa \\ Email: wduplessis@ieee.org \\ ${ }^{*}$ King Abdulaziz City for Science and Technology (KACST) \\ Riyadh, Saudi Arabia
}

\begin{abstract}
The effect of allowing shared elements in interleaved thinned arrays is investigated. The sidelobe level (SLL) as a function of the number of shared elements mirrors the SLL of thinned arrays as a function of filling factor because the number of shared elements determines the filling factor of the subarrays. When the number of shared elements is small, the result is two subarrays on opposite sides of the aperture with only their ends being interleaved.
\end{abstract}

\section{INTRODUCTION}

The sharing of antenna apertures is becoming increasingly important as a result of the combination of limited available antenna real estate and the requirement for large numbers of sensor and communication systems on modern platforms [1]. Interleaved thinned arrays achieve aperture sharing by allowing multiple thinned arrays (subarrays) to share an aperture with each subarray having a different function.

Interleaved thinned arrays have recently attracted interest in the literature (e.g. [1]-[4]) due to their ability to realise aperture sharing while maintaining good antenna properties. Some examples include the use of differently-sized antenna elements [2] operating over different frequency ranges, decreasing leakage between the transmitter and receiver of a continuous-wave (CW) radar [3] and achieving multiple polarisations with single-polarised antenna elements [4].

The main published methods for the synthesis of interleaved thinned arrays are genetic algorithms (GAs) [1], [4] and cyclic difference sets [2], [3], [5]. In these cases, each subarray is the inverse of the other (each element is allocated to only one of the subarrays). This approach has a number of benefits including simplified construction of the resulting array because each element only has one function and a simplification of the synthesis process through a reduction of the available degrees of freedom. However, there are reasons to believe that allowing elements to be shared will lead to improved performance.

The best SLL performance for thinned arrays without interleaving is achieved when the active elements of the array are concentrated near the centre of the array [6]-[8]. The densitytaper algorithm is based on the fact that the ensemble average of a large number of patterns where the active elements are selected according to an underlying real distribution is the

This work was supported by Advanced Sensors and Electronic Defence (ASED), a centre of the King Abdulaziz City for Science and Technology (KACST). pattern achieved by the distribution [6]. Given that distributions which achieve good sidelobe performance have larger excitations near the centre of the array [9] and thus higher probabilities of selecting those elements, this provides a theoretical motivation for favouring the central elements in thinned arrays. Algorithmically-synthesised thinned arrays support this theoretical observation (e.g. [7], [8]). The interleaved thinnedarray synthesis techniques highlighted above cannot achieve high element densities near the centre of the aperture for all the subarrays because each element can only belong to one subarray.

The best SLL values for thinned arrays without interleaving are achieved when the proportion of the elements which is active (the filling factor) is on the order of $85 \%$ for small- to medium-sized arrays [10], though values in the range of $75 \%$ to $80 \%$ appear to give the best results for larger arrays [7], [10]. Again, the interleaved thinned-array synthesis techniques mentioned above cannot achieve these desirable filling-factor values because the filling factor will always be very close to $50 \%$ as a result of the requirement that each element be part of only one subarray.

This paper explores the effect of allowing elements of interleaved thinned arrays to be shared by more than one subarray. Based on the discussion above, it is expected that this will lead to improved SLL because higher element densities are possible near the centre of the shared aperture and higher filling factors can be achieved for each subarray.

The increased system complexity entailed by allowing subarrays to share elements will depend strongly on the application of the antenna. For example, shared elements will be more difficult to implement in CW radar systems than in pulsed radar systems. In either case, the SLL improvement that can be achieved will have to be weighed against the additional complexity entailed by sharing elements. This paper seeks to aid such evaluations by considering the relationship between the SLL and the number of shared elements.

\section{Algorithm Used to Generate Results}

The GA [11] utilised to generate the results presented in Section III is described below and summarised in Fig. 1. GAs mimic evolution and natural selection in order to obtain improved solutions and have been successfully applied to a wide variety of problems. These problems include thinned- 


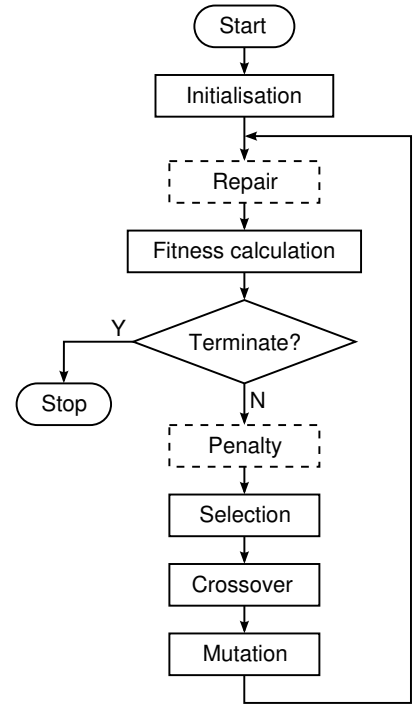

Fig. 1. A flowchart of the GA used to generate the results presented in Section III. The different outlines of the repair and penalty blocks are to emphasise the fact that they are not always used.

and interleaved-array synthesis [1], [7], suggesting that a GA is the appropriate choice for this implementation.

The population size was chosen to be large because larger populations were found to give better solutions. However, the population size was limited by the time available to run the algorithm with on the order of $90 \mathrm{~s}$ being required per run on a quad-core Intel Core i7 CPU running at $2.20 \mathrm{GHz}$.

The number of genes in each chromosome was twice the number of elements in the aperture, with the first and second halves of each individual representing the two subarrays This representation allows all possible combinations of two interleaved subarrays to be represented.

The algorithm was initialised by assigning each gene a value of 0 or 1 with equal probability.

The SLL achieved by each solution was used as the fitness. The array pattern was computed from the inverse fast Fourier transform (IFFT) with 2,048 points, which is approximately 20 points per pattern root in line with other published results (e.g. [8]). The pattern of the best result was computed with 32,768 points to ensure highly accurate results. The SLL was then determined from the level of the highest peak relative the main beam.

The GA was run for a fixed number of generations to eliminate the need for a complex termination test in line with common practice [11]. The number of generations was chosen to be large enough to ensure that improvements in the best solution were extremely rare when additional generations were added.

Elitism was implemented to ensure monotonic convergence. Elitism is the process of copying the best solution from the previous generation to the new generation to ensure that the best solution is never lost in the process of creating a new generation and discarding the old one [11].

Exponential-ranking selection was utilised, selecting the individuals used to create the next generation according to

\begin{tabular}{l|llllllll} 
Parent 1: & $\mathbf{0}$ & 1 & $\mathbf{0}$ & 1 & 0 & 1 & $\mathbf{1}$ & $\mathbf{1}$ \\
Parent 2: & 0 & $\mathbf{0}$ & 1 & $\mathbf{1}$ & $\mathbf{1}$ & $\mathbf{0}$ & 0 & 1 \\
\hline Selection: & 1 & 2 & 1 & 2 & 2 & 2 & 1 & 1 \\
\hline Offspring: & 0 & 0 & 0 & 1 & 1 & 0 & 1 & 1
\end{tabular}

Fig. 2. An example of uniform crossover.

the following relationship [12]

$$
\frac{P_{n+1}}{P_{n}}=\frac{P_{n}}{P_{n-1}}
$$

where $P_{n}$ is the probability of selecting individual $n$ and the solutions are ranked so that larger $n$ means better SLL. This selection scheme was chosen because fine control of the bias towards better solutions is achieved with a single parameter.

Uniform crossover was used generate new candidate solutions by combining existing solutions. As shown in Fig. 2, the genes in a new chromosome are created by randomly selecting between the genes of the two parents [11]. The main benefit of uniform crossover is that the ordering of the genes in a chromosome has a much smaller effect than for normal crossover. Crossover is only used to generate a specified proportion of the new population, and the remainder of the new population is generated by simply copying an existing solution.

Crossover was followed by mutation, during which a small number of randomly-selected genes in the population were inverted [11]. Mutation is required to reduce the likelihood that the GA will become trapped in a locally-optimal solution.

The process was then repeated starting with fitness evaluation as shown in Fig. 1. Each cycle through the loop is known as a generation because a new population is created.

The results in Section III was generated using a population of 5,000 individuals run for 150 generations with crossover and mutation probabilities of 0.9 and 0.01 respectively. The selection parameter $(\kappa)$ was 0.0005 . The GA was run twenty times and the results with the best SLL values were used. This was done in an attempt to reduce the effects of the algorithm and thereby emphasise the inherent properties of interleaved thinned arrays with shared elements.

While the GA described above is extremely useful and gives excellent results for a number of problems, it does not allow control of the number of shared antenna elements. Three possibilities to achieve such control are:

- a penalty function,

- a repair algorithm, and

- developing a custom GA where all solutions inherently satisfy the constraints.

While the last option is preferable [13], it is unclear how the GA could be modified to achieve a specified number of shared elements.

Penalty functions are widely used in constrained optimisation [14], and work by artificially degrading a fitness of a solution (penalising the solution) if it does not satisfy a constraint. In this case, the constraint is the required number of shared elements, so penalty was computed using

$$
P=20\left|O-O_{s}\right| \frac{G}{G_{x}}
$$




\begin{tabular}{l|llll|lll|llll} 
& \multicolumn{4}{|c|}{ Not shared } & \multicolumn{3}{|c|}{ Shared } & \multicolumn{4}{c}{ Not shared } \\
\hline Subarray 1: & 1 & 0 & 0 & 0 & 1 & 1 & 1 & 1 & 0 & 0 & 1 \\
Subarray 2: & 0 & 1 & 1 & 0 & 1 & 1 & 1 & 0 & 1 & 0 & 0
\end{tabular}

Fig. 3. An example of the output of the implemented repair algorithm for an eleven-element aperture with three shared elements.

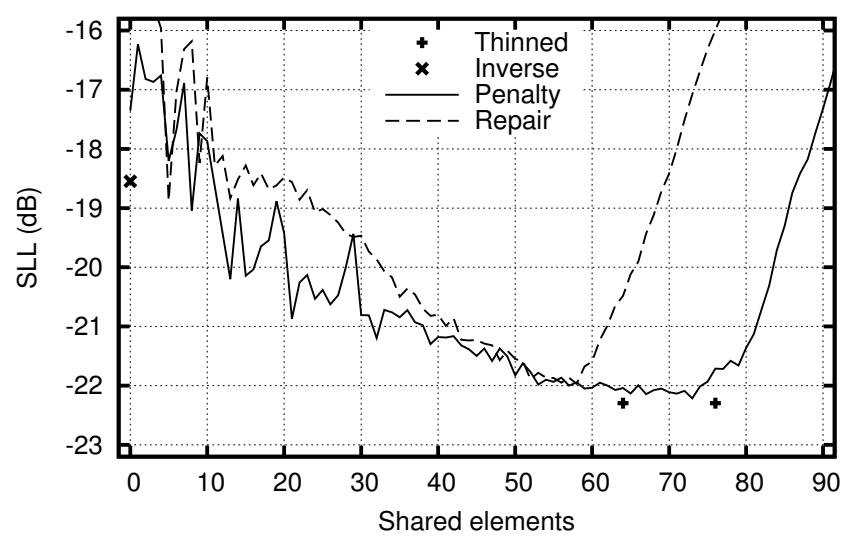

Fig. 4. SLL as a function of the number of shared elements for 100-element interleaved linear arrays with two subarrays. The filling factors are plotted in Fig. 5 and selected excitations are provided in Table II.

where $P$ is the penalty in decibels, $O$ and $O_{s}$ are the achieved and specified overlap respectively and $G$ and $G_{x}$ are the current and maximum number of generations respectively.

Repair algorithms modify unsuitable solutions in such a way that they satisfy the constraints. For example, a parameter value that is greater than the allowable maximum would be changed to be equal to the specified maximum. As outlined in Section I, it is reasonable to assume that the central elements of an array are most likely to be shared between interleaved thinned subarrays. The implemented repair algorithm ensured that the specified number of elements was shared by activating that number of elements at the centre of the array and ensuring that no other elements were shared as shown in Fig. 3. The main benefit of this approach is that the number of elements which the GA must optimise is reduced because the central elements are always active and are thus not subject to optimisation.

\section{Results ObTAINED AND Discussion}

Fig. 4 shows the best SLL obtained as a function of the number of shared elements. The points associated with a uniformly-excited array, a single thinned array and an interleaved array with inverse subarrays as in [1]-[4] are shown. Two thinned-array cases are shown: the case where the subarrays are identical, and the case where one of the subarrays is flipped.

The fact that the best results for interleaved thinned arrays are within $0.01 \mathrm{~dB}$ of the best results for a single thinned array despite the fact that the thinned array only has 100 unknowns while the interleaved case has 200 variables. This suggests that the GA described in Section II performs well when the number of shared elements is high.

However, the results with no overlap are significantly worse than the results where one subarray is the inverse of the

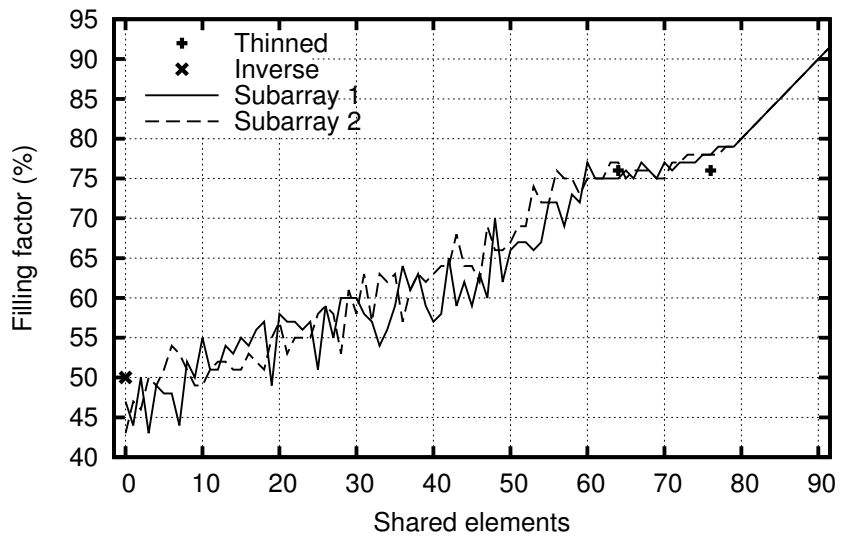

Fig. 5. The filling factor as a function of the number of shared elements for the penalty-function GA results in Fig. 4

other. Additionally, there is a large variation in the results for a small number of shared elements which reduces as the number of times the GA is run increases. Taken together, these points suggest that the GA implemented here does not provide consistently good results when the number of shared elements is small. This point will be explored further below in connection with subarray excitations.

Fig. 4 shows that the results achieved by the penaltyfunction approach are usually better than those achieved by the repair-algorithm approach. The main exception is when the number of shared elements is between roughly 40 and 60 where the results are similar. Only allowing the central elements to be shared does thus not lead to good results for large and small numbers of shared elements. This suggests that the effect of shared edge elements is significant and cannot be ignored. Given that the penalty-function results are at least as good as the repair-algorithm results, only the penalty-function results will be considered below.

The first important observation about the underlying properties of interleaved thinned arrays is that the best SLL initially tends to improve as the number of shared elements increases. A turning point is then reached after which the SLL rapidly deteriorates as the number of shared elements increases. These trends are expected based on similar trends observed for thinned arrays [7], [10] where an optimal filling factor is observed.

The discussion now naturally moves to the relationship between the number of shared elements and the filling factor as a way to reconcile known results for thinned arrays with the results obtained here. Fig. 5 plots the desired relationship.

The filling factor for the two subarrays is always similar. This is reasonable given that similar SLL performance is required from both subarrays because the SLL of the worse subarray is the desired performance metric.

The filling factor initially increases approximately linearly showing that allowing more shared elements leads to an increased filling factor for the subarrays. This observation is quite reasonable in light of the fact that the number of available elements is limited.

Between 60 and 75 shared elements, the filling factor remains nearly constant in the range of $75 \%$ to $80 \%$. The 
thinned-array result with the smallest overlap is within this region (64 shared elements, $76 \%$ filling factor). Furthermore, filling factors of $75 \%$ to $80 \%$ have been shown to give good results for single thinned arrays [7], [10]. The excellent SLL results from 60 to 75 shared elements in Fig. 4 can thus explained by the known properties of thinned arrays.

Finally, the filling factors and the number of shared elements are equal for 79 or more shared elements. This rather noticeable change corresponds to a rapid deterioration of the SLL in Fig. 4.

The excitations for a number of shared element values are presented in Table I. The excitations of the inverse case and the single thinned array case with one of the excitations flipped are also shown in Table I for comparison purposes.

Table I shows that the overwhelming majority of the shared elements are near the centre of the array apertures. This mirrors the case for thinned arrays where the majority of the active elements are near the centre of the aperture [6]-[8] However, the shared elements are not limited to the centre of the aperture, and as stated previously in connection with the performance of the repair algorithm, shared elements near the edges of the aperture have a significant effect.

The excitations of the two subarrays are identical when the number of shared elements is large ( 80 or more). This region corresponds to a rapid deterioration of the SLL in Fig. 4 and the rightmost portion of Fig. 5 where the number of shared elements is equal to the subarray filling factors. The small number of inactive elements combined with the large number of shared elements make this situation unavoidable because only a very small number of good solutions exists.

Examining the cases for smaller numbers of shared elements reveals some interesting trends. Firstly, the inverse case achieves significantly better results than the more general shared-element case considered here. This is particularly surprising given that an inverse interleaved array is a special case of the interleaved thinned array considered here. Secondly, as shown in Fig. 4, the SLL values vary dramatically for minimal changes in the number of shared elements.

The fact that the inverse case gives better results than the shared-element case is at least partially attributable to the fact that the inverse case only has 100 binary variables while the shared-element case has 200 variables. This means that the shared-element case has $2^{100}$ times more possible solutions, greatly complicating the problem.

However, the most interesting observation for interleaved arrays with small numbers of shared elements is that the active elements of each subarray tend to be concentrated on one side of the available aperture. This is particularly noticeable for the inverse case and for 8 shared elements (the solution with the best SLL for less than 12 shared elements). This suggests that the ideal layout for interleaved thinned arrays with few shared elements comprises subarrays on opposite sides of the aperture which only share the elements at the extreme ends of their apertures. The partially-interleaved arrays considered in [1] are similar to this approach, but the current approach is far more general because shared elements are allowed and the interleaved region is determined algorithmically.

The hypothesis that the arrays should be concentrated on

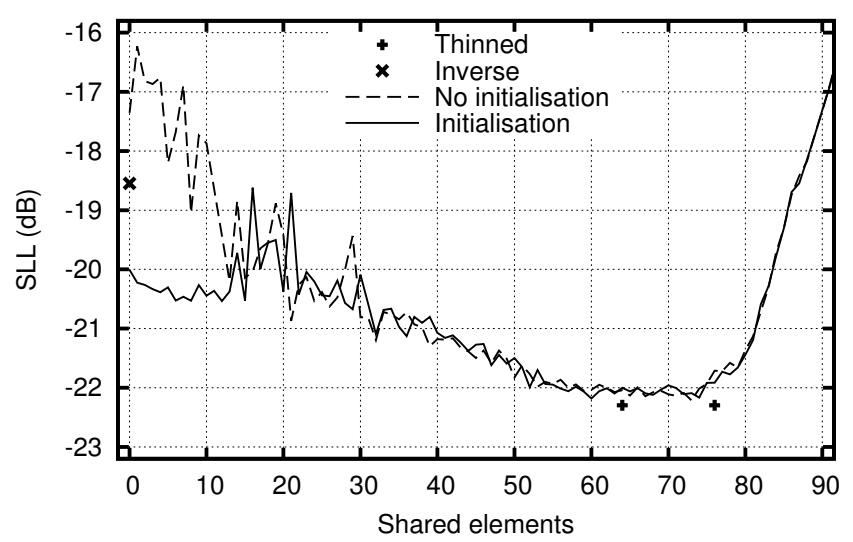

Fig. 6. The SLL as a function of the number of shared elements for the penalty-function implementation both with and without initialisation. Selected excitations are provided in Table II.

opposite sides of the aperture was investigated by re-running the penalty-function GA with a single individual initialised to the non-interleaved case shown in Table II. The results obtained are shown in Fig. 6 and some excitations are provided in Table II.

Despite the fact that only one individual of 5,000 in the initial population was modified, a remarkable improvement in the SLL values for small numbers of shared elements is seen in Fig. 6. The hypothesis is thus to be validated by the large SLL improvement despite the minor change to the GA.

A further reason for the large improvement over the inverse case is the fact that not all the elements are active. This improves the SLL by allowing the element density to be tapered towards the edges of each subarray as seen in Table II.

The initialisation does not have a significant effect for larger numbers of shared elements. A significant SLL improvement is only achieved for less than thirteen shared elements, showing that the effect of the initialisation is negligible for more than thirteen shared elements. The excitations in Table I show that this is reasonable because the subarrays for larger numbers of shared elements tend to share the centre of the aperture rather than being concentrated on opposite sides of the array.

\section{CONCLUSION}

The effect of allowing elements to be shared between subarrays of interleaved thinned arrays is investigated. A GA with either a penalty function or a repair algorithm was used to synthesise results for thinned arrays with the number of shared elements being varied from 0 to $90 \%$ of the total number of elements.

The main conclusion of this work is that the known properties of thinned arrays are closely mirrored by interleaved thinned arrays with shared elements. Most importantly, the filling factor is closely related to the number of shared elements, so the existence of an optimal filling factor is mirrored by the existence of an optimal number of shared elements.

Another important conclusion is that the subarrays tend to have the majority of their active elements on opposite sides of the aperture when the number of shared elements is small. This means that interleaving only occurs for the outermost elements 
TABLE I

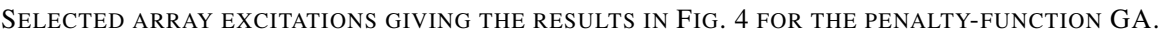

\begin{tabular}{|c|c|c|}
\hline Description & ubarray & Excitation \\
\hline \multirow{2}{*}{ Inverse } & 1 & 1111111111111111111111111111111111111111011010101100101010010000000000000000000000000000000000000000 \\
\hline & & 0000000000000000000000000000000000000001001010100110101011011111111111111111111111111111111111111111 \\
\hline \multirow{2}{*}{ Shared: 0} & 1 & 00111111111111111111111110111111101101011110000011101001110000000010000100000000000000000000000000 \\
\hline & 2 & 0000000000000000000000000010000000000101000001100000100100011111111011110111111111111111110111111101 \\
\hline \multirow{2}{*}{ Shared: 8} & 1 & \\
\hline & 2 & 11001111111101111111111111111111111011110111111010110010101000000001000000000000000000000000000000 \\
\hline \multirow{2}{*}{ Shared: 10} & 1 & 0111111111101111111111011111101111110111111110100110010110010001000010100100001000100000001000000010 \\
\hline & 2 & 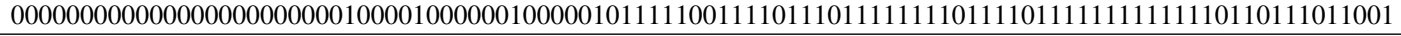 \\
\hline \multirow[b]{2}{*}{ Shared: 20} & 1 & \\
\hline & 2 & 000000 \\
\hline \multirow{2}{*}{ Shared: 30} & 1 & 000 \\
\hline & 2 & 011 \\
\hline \multirow{2}{*}{ Shared: 40} & 1 & 000 \\
\hline & 2 & 10001001000000000000 \\
\hline \multirow[b]{2}{*}{ Shared: 50} & 1 & 000 \\
\hline & 2 & 00000 \\
\hline \multirow{2}{*}{ Shared: 60} & 1 & 10000011010 \\
\hline & 2 & \\
\hline \multirow{2}{*}{ Thinned: 64} & 1 & 11010000001001000111100111111111111111111111111111111111111111111111111111111111010101110100101001101 \\
\hline & 2 & 101100101001011101010111111111111111111111111111111111111111111111111111111111100111100010010000001011 \\
\hline \multirow{2}{*}{ Shared: 70} & 1 & $\overline{00000110}$ \\
\hline & 2 & \\
\hline \multirow{2}{*}{ Thinned: 76} & 1 & 11010000001001000111100111111111111111111111111 \\
\hline & 2 & 11010000001001000111100111111111111111111111111111111111111111111 \\
\hline \multirow[b]{2}{*}{ Shared: 80} & 1 & \\
\hline & 2 & \\
\hline \multirow{2}{*}{ Shared: 90} & 1 & 1111111111001001111011111111111111111111111111111111111111111111111111111111111111111010110100111111111 \\
\hline & 2 & 1111111111001001111011111111111111111111111111111111111111111111111111111111111111010110100111111111 \\
\hline
\end{tabular}

TABLE II

SELECTED ARRAY EXCITATIONS GIVING THE RESULTS IN FIG. 6 FOR THE PENALTY-FUNCTION GA WITH NON-INTERLEAVED INITIALISATION.

\begin{tabular}{lc|c}
\hline \multicolumn{1}{c}{ Description } & Subarray & Excitation \\
\hline $\begin{array}{l}\text { Non- } \\
\text { interleaved }\end{array}$ & 1 & 1111111111111111111111111111111111111111111111111100000000000000000000000000000000000000000000000000 \\
\hline \multirow{2}{*}{ Inverse } & 1 & 00000000000000000000000000000000000000000000000000111111111111111111111111111111111111111111111111 \\
\hline \multirow{2}{*}{ Shared: 0} & 2 & 11111111111111111111111111111111111111011010101100101010010000000000000000000000000000000000000000 \\
& 2 & 1110000000000000000000000000000000000010010101001101010110111111111111111111111111111111111111111 \\
\hline \multirow{2}{*}{ Shared: 5} & 1 & 0000000000000000000000000000000000000000000000000011110001010000000000000000000000000000000000000000 \\
& 2 & 0000000000000000000000000000000000000000011111111111111111111111111111110110010 \\
\hline \multirow{2}{*}{ Shared: 10} & 1 & 1111100111011111111111111111111111111111111111101101101000000111111111111111111111111111111111111110011010110 \\
& 2 & 00000000000000000000000000000000001001000100110101100111111111111111111111111111111111111101111010111 \\
\hline
\end{tabular}

of each subarray, suggesting that the value of interleaving and/or sharing elements is small unless a large number of elements is shared.

The results presented here are subject to the following limitations which will be addressed in future work:

- only a 100-element aperture was considered,

- only a linear array was considered,

- only SLL was evaluated, and

- only two subarrays were considered.

\section{REFERENCES}

[1] R. L. Haupt, "Interleaved thinned linear arrays," IEEE Trans. Antennas Propag., vol. 53, no. 9, pp. 2858-2864, September 2005.

[2] C. I. Coman, I. E. Lager, and L. P. Ligthart, "The design of sharedaperture antennas consisting of differently sized elements," IEEE Trans. Antennas Propag., vol. 54, no. 2, pp. 379-383, February 2006.

[3] I. E. Lager, C. Trampuz, M. Simeoni, and L. P. Ligthart, "Interleaved array antennas for FMCW radar applications," IEEE Trans. Antennas Propag., vol. 57, no. 8, pp. 2486-2490, Aug. 2009.

[4] R. Guinvarc'h and R. L. Haupt, "Dual polarization interleaved spiral antenna phased array with an octave bandwidth," IEEE Trans. Antennas Propag., vol. 58, no. 2, pp. 397-403, Feb. 2010.
[5] D. G. Leeper, "Isophoric arrays - massively thinned phased arrays with well-controlled sidelobes," IEEE Trans. Antennas Propag., vol. 47, no. 12 , pp. 1825-1835, December 1999.

[6] M. I. Skolnik, J. W. Sherman, III, and F. C. Ogg, Jr, "Statistically designed density-tapered arrays," IEEE Trans. Antennas Propag., vol. 12, no. 4, pp. 408-417, July 1964.

[7] R. L. Haupt, "Thinned arrays using genetic algorithms," IEEE Trans. Antennas Propag., vol. 42, no. 7, pp. 993-999, July 1994.

[8] W. P. M. N. Keizer, "Linear array thinning using iterative FFT techniques," IEEE Trans. Antennas Propag., vol. 56, no. 8, pp. 2757-2760, August 2008.

[9] F. J. Harris, "On the use of windows for harmonic analysis with the discrete Fourier transform," Proc. IEEE, vol. 66, no. 1, pp. 51-83, January 1978 .

[10] J. W. Hooker and R. K. Arora, "Optimal thinning levels in linear arrays,' IEEE Antennas Wirel. Propag. Lett., vol. 9, pp. 771-774, 2010.

[11] D. E. Goldberg, Genetic algorithms in search, optimization, and machine learning. Addison-Wesley, 1989

[12] T. Blickle and L. Thiele, "A comparison of selection schemes used in evolutionary algorithms," Evolutionary Computation, vol. 4, no. 4, pp. 361-394, 1996.

[13] Z. Michalewicz, Genetic algorithms + data structures = evolution programs. Springer-Verlag, 1992.

[14] J. Nocedal and S. J. Wright, Numerical optimization. Springer, 1999. 